\title{
Software for Machine-Independent Quantitative Interpretation of SSCP in Capillary Array Electrophoresis (QUISCA)
}

BioTechniques 33:1342-1348 (December 2002)

\author{
Koichiro Higasa ${ }^{1}$, Yoji \\ Kukita $^{1,2}$, Shingo Baba ${ }^{1}$, \\ and Kenshi Hayashi ${ }^{1}$ \\ ${ }^{1}$ Kyushu University, Fukuoka, \\ and ${ }^{2}$ University of Tokyo, \\ Tokyo, Japan
}

\begin{abstract}
PCR single-stranded conformational polymorphism (SSCP) analysis is a simple and rapid electrophoretic technique for the sensitive detection of sequence variants of PCR products. Here we describe a crossplatform program package, quantitative interpretation of SSCP in capillary array (QUISCA), which allows semi-automated quantitative detection of sequence variants separated by multicolor fluorescence-based SSCP electrophoresis using various capillary array apparatus. The program, together with the QUISCAview as a graphical user interface, takes trace data in ASCII format and processes them with three modules: signal denoising/baseline subtraction, color-matrix construction/application, and calibration of peak positions between multiple capillary runs using internal standard peaks. QUISCA is compatible with data from various widely used capillary array sequencers and is suitable not only for finding or typing SNPs in individual DNAs but also for the accurate estimation of the allele frequencies of many SNPs using a pooled DNA strategy. QUISCA can also serve as a versatile core program for various fragment analyses.
\end{abstract}

\section{INTRODUCTION}

With the approaching completion of the production of a reference human genome sequence, the next important target will be the extensive characterization of variations in the genomes of individuals. Ubiquitous occurrence of SNPs in the genome $(11,15)$ has prompted academic and pharmacogenomic communities to collect them for use as genetic markers in genome-wide association studies to identify the genes responsible for polygenic traits, such as disease susceptibility or sensitivity/resistance to drugs. Because of the number of SNPs and even higher numbers of potential samples that need to be characterized in the association study $(5,10)$, the development of a widely accessible method suitable for high throughput and low-cost analysis of SNPs is desirable.

Recently, we developed a method for SNP characterization, in which PCR products are post-labeled with fluorescent dyes and analyzed directly with widely available automated capillary electrophoresis DNA sequencers under conditions for single-stranded conformational polymorphism (SSCP) analysis (PLACE-SSCP) $(3,4)$. This method is suitable not only for the determination of SNP genotypes of individuals at a low cost but also for the estimation of the allele frequencies using pooled DNA at a high precision (12).

We have extended this method to a multichannel capillary electrophoresis system to increase the throughput (6). During the course of establishing this paralleled analysis system, we found it necessary to develop software suitable for the analysis.

In this report, we present a program that allows for the processing of raw trace data of SSCP analysis, obtained from various capillary-based electrophoresis systems, to obtain peak information such as peak identity and height. This software has been extensively tested in our high-throughput SNP quantification project that involved the SSCP analysis of SNPs using a variety of commercially available capillary-based DNA sequencers.

\section{MATERIALS AND METHODS}

\section{Algorithm Overview}

Figure 1 shows the flowchart for data processing using the quantitative interpretation of SSCP in capillary array electrophoresis (QUISCA). The functions implemented in QUISCA are noise filtering, baseline subtraction, color separation, peak detection, global data point calibration (alignment), and color-matrix construction. The data processed in each step of QUISCA are exported to an interface, QUISCAview, and visualized to assist operation. Other functions implemented at this interface are local peak detection, peak selection, and alignment. 


\section{Data Collection and Preparation of Input Files}

The input file for QUISCA is a multi-column, multi-line fluorescence intensity table in ASCII format (trace file), in which columns and lines represent the preset wavelength ranges and the time points for data collection, respectively. One trace file is generated for each lane of the capillary array. The fluorescence data file of PLACE-SSCP obtained from an ABI PRISM ${ }^{\circledR} 3100$ Genetic Analyzer or 3700 DNA Analyzer is converted to a trace file using a Tool Kit (all from Applied Biosystems, Foster City, CA, USA). The data from the MegaBACE ${ }^{\mathrm{TM}}-1000$ DNA Analysis System is exported as a trace file using MegaBACE Sequence Analysis Software (both from Amersham Biosciences, Piscataway, NJ, USA).

\section{Noise Filtering}

Noise can originate from several sources, including the thermal noise of the detector and other noises from the operating environment. We have experimented with several types of noise-removal techniques (13) and found a satisfactory filtration using the Fast Fourier Transform (FFT) that removes high-frequency noise (9).

The filtering process proceeds as follows for each channel of raw data. First, the data are transformed to the frequency domain by means of an FFT. The resulting complex array is then multiplied point by point against the filter function below, and finally the inverse FFT is applied to transform the filtered data in the frequency domain back to the time domain. The filter is a quadratic function described by:

$\begin{array}{lll}F(f)=1-D^{2} f^{2}, & \text { if } & F(f) \geq 0 \\ F(f)=0, & \text { if } & F(f)<0\end{array}$

where $\mathrm{f}$ is the frequency, and $\mathrm{D}$ is the determinant of the high-frequency cutoff. Parameter D is chosen on the basis of the sampling rate and the amount of noise, as detailed in the following section. The filter allows a gradual highfrequency cut-off.

\section{Baseline Subtraction}

The background signal in raw data differs among channels (wavelength ranges) and capillaries and changes slowly with time. Baseline subtraction places the background signal of all traces of the electropherogram at zero, which is a necessary step for the accurate quantification of the peaks and for easier visual identification of the features of the peaks.

In QUISCA, baseline subtraction starts by searching for the minimum signal intensity in successive windows of size $\mathrm{N}$, where $\mathrm{N}$ is user selectable. The baseline is estimated by the linear interpolation between the neighboring minima. Signal intensities at each data point are corrected by subtracting the corresponding values of the estimated baseline.

\section{Color Separation}

The fluorophores used in the multidye capillary electrophoresis system have distinct emission spectra. Color separation uses a spectral cross-talk matrix (M) to remove the unwanted signals from the targeted dyes in each data channel. This transformation is performed by $\mathrm{f}=\mathrm{Md}$, where $\mathrm{f}$ represents the vector of fluorescence intensity measured at each wavelength range (in "fluorescence space"), and d is the vector in "dye space." For our purposes, it is necessary to perform the inverse operation, converting f to $\mathrm{d}$. To do this, $\mathrm{M}$ is inverted numerically (9) and used in the equation $d=M^{-1} f$.

In default, QUISCA is set to deal with four dyes. A $4 \times 4$ spectral crosstalk matrix is constructed by running four kinds of color standard samples separately and determining fluorescence intensities at four preset wavelength ranges, followed by normalization. This matrix is stored and then used by QUISCA for the data from MegaBACE. Trace data from an ABI PRISM 3100 or 3700 Analyzer are already color-separated within the ABI PrISM Data Collection Software (Applied Biosystems) so that the diagonal matrix is used.
Figure 1. Flowchart of QUISCA/QUISCAview. The QUISCA program was designed to accept multispectral trace data collected on a fluorescence-based capillary array electrophoresis apparatus. The program processes the data through a set of processing algorithms and then allows these data to be saved. Each set of processed data is viewed with a graphical user interface, QUISCAview, in which additional functions, including local alignment and peak calling, are also implemented. 


\section{Peak Detection}

Peaks are sought by scanning the trace for regions that are convex; that is, $\mathrm{p}^{\prime}(\mathrm{t}) \geq 0$ and $\mathrm{p}^{\prime}(\mathrm{t}+1)<0$, where $\mathrm{p}^{\prime}(\mathrm{t})$ is the first derivative of the trace data at time point $t$. The peaks that have values of height and width less than the thresholds (h and w, respectively) are then rejected. Here peak width is defined by the distance (in time points) between the nearest time points where $\mathrm{p}^{\prime}(\mathrm{t}) \leq 0$ and $\mathrm{p}^{\prime}(\mathrm{t}+1)>0$.

\section{Alignment}

The purpose of this process is to correct capillary-to-capillary or run-to-run variation in mobilities and to aid peak identification and quantification. This process evaluates the heights and positions of peaks for internal reference markers that are labeled with a dye different from those of the samples and adjusts the mobility variation either globally or locally within a given range selected by the user. The associated

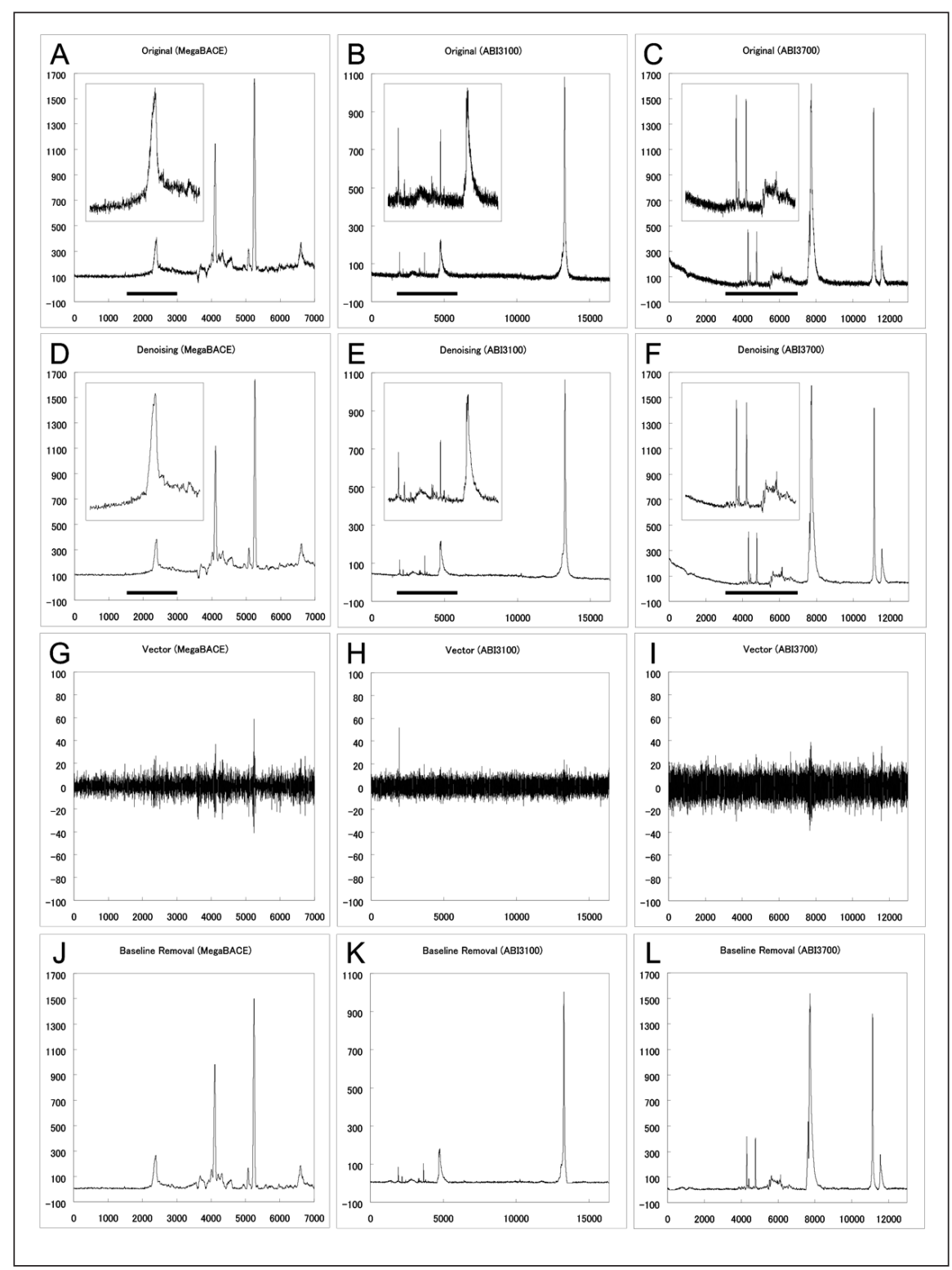

Figure 2. Noise filtering and baseline adjustment. (A-C) Original signals from three apparatus, the MegaBACE (left), ABI PRISM 3100 Genetic Analyzer (middle), and ABI PRISM 3700 DNA Analyzer (right). (D-F) Reconstructed signals after filtering. (G-I) Vector of differences between the filtered and original signals. (J-L) Processed signals after filtering and baseline removal. Bars in panels A-F indicate the regions of traces expanded in the insert panels. software, QUISCAview, performs the local alignment.

The global alignment consists of three processes: the detection of reference peaks; assignment of these peaks in each run (sample run) to the corresponding peaks in a preselected template run; and calibration of the time points of sample peaks in sample runs. The algorithm for peak detection is described in the previous section. Peak assignment is the most complex part of the alignment process. The difficulty in identifying each marker peak in different runs comes from various sources, such as peak height variability, noise, poor peak resolution, and peak spacing anomalies. In comparing the data between runs, the heights and positions of individual peaks are often inconsistent, but their relative values (i.e., the peak pattern) are usually similar. In QUISCA, the data of each sample run are divided into blocks that are delimited by the two neighboring peaks. Then, the most likely start positions of the $\mathrm{n}^{\text {th }}$ block in the template run, $t_{n}$, is adaptively determined starting from the first block. This is done by evaluating $\mathrm{Q}(\mathrm{t})$, which is given by the following equation:

$$
\begin{aligned}
& \mathrm{Q}(\mathrm{t})= \sum_{\mathrm{i}=-\mathrm{r}}^{\mathrm{B}_{\mathrm{n}}+\mathrm{r}}\left|\mathrm{T}(\mathrm{t}+\mathrm{i})-\mathrm{S}\left(\mathrm{S}_{\mathrm{n}}+\mathrm{i}\right)\right|, \\
& \mathrm{t}_{\mathrm{n}-1}+\mathrm{B}_{\mathrm{n}-1}-\mathrm{R} \leq \mathrm{t} \leq \mathrm{t}_{\mathrm{n}-1}+\mathrm{B}_{\mathrm{n}-1}+\mathrm{R}
\end{aligned}
$$

where $T(t)$ and $S(s)$ are the fluorescence intensities of traces at time points $t$ and $s$ of template and sample runs, respectively. $B_{n}$ is the width of the $n^{\text {th }}$ block, $s_{n}$ is the starting position of the $\mathrm{n}^{\text {th }}$ block in each run, $\mathrm{r}$ is the block margin needed to optimize the search, and $\mathrm{R}$ is the range of the search. Then, $t_{n}$ is obtained as a value of $t$ that satisfies following equation:

$\mathrm{Q}\left(\mathrm{t}_{\mathrm{n}}\right)=\min \mathrm{Q}(\mathrm{t})$

After the relative positions of similar patterns have been found, the peaks in a template run that correspond to the peaks in the sample runs are identified by the similarity of their positions within the appropriate blocks. Finally, all data points in sample runs are calibrated by one of two algorithms: either cubic spline (9) or the local Southern (14) interpolation method. 
Table 1. Mixing Experiment Data Processed by QUISCA and GeneScan Analysis Software

\begin{tabular}{|c|c|c|c|c|c|c|c|c|}
\hline \multirow{3}{*}{$\begin{array}{l}\text { Percent of } \\
\text { Allele } 1\end{array}$} & \multicolumn{4}{|c|}{ QUISCA } & \multicolumn{4}{|c|}{ GeneScana } \\
\hline & \multicolumn{2}{|c|}{ Peak Heights ${ }^{b}$} & \multicolumn{2}{|c|}{ Fraction of Allele 1} & \multicolumn{2}{|c|}{ Peak Heights ${ }^{b}$} & \multicolumn{2}{|c|}{ Fraction of Allele 1} \\
\hline & Allele 1 & Allele 2 & Rawc & Correctedd & Allele 1 & Allele 2 & Rawc & Correctedd \\
\hline 0 & 0 & 3577 & 0.0 & 0.0 & 0 & 3546 & 0.0 & 0.0 \\
\hline 10 & 402 & 2937 & 12.0 & 9.6 & 392 & 2915 & 11.9 & 9.6 \\
\hline 20 & 834 & 2524 & 24.8 & 20.5 & 833 & 2525 & 24.8 & 20.7 \\
\hline 30 & 1158 & 2122 & 35.3 & 29.8 & 1179 & 2133 & 35.6 & 30.4 \\
\hline 40 & 1579 & 1820 & 46.5 & 40.3 & 1577 & 1825 & 46.4 & 40.6 \\
\hline 50 & 1426 & 1111 & 56.2 & 50.0 & 1434 & 1132 & 55.9 & 50.0 \\
\hline 60 & 2313 & 1164 & 66.5 & 60.8 & 2319 & 1174 & 66.4 & 60.9 \\
\hline 70 & 2558 & 887 & 74.3 & 69.2 & 2582 & 905 & 74.0 & 69.3 \\
\hline 80 & 2901 & 586 & 83.2 & 79.4 & 2899 & 596 & 82.9 & 79.3 \\
\hline 90 & 3175 & 295 & 91.5 & 89.3 & 3171 & 300 & 91.4 & 89.3 \\
\hline 100 & 3159 & 0 & 100.0 & 100.0 & 3160 & 0 & 100.0 & 100.0 \\
\hline $\begin{array}{l}\text { aThese data } \\
\text { bPeak heigh } \\
\text { cPercent all } \\
\text { dPercent all } \\
\text { Reference }\end{array}$ & $\begin{array}{l}\text { re taken fr } \\
\text { in arbitrar } \\
1 \text { estimat } \\
1 \text { estima }\end{array}$ & $\begin{array}{l}\text { om Sasak } \\
\text { y units. } \\
\text { ed from } p \\
\text { ted from c }\end{array}$ & $\begin{array}{l}\text { 12). } \\
\text { phts in ra } \\
\text { peak he }\end{array}$ & $\begin{array}{l}\text { N data. } \\
\text { ights. Details }\end{array}$ & orrection & ethod & cribed $k$ & \\
\hline
\end{tabular}

The local alignment is carried out by QUISCAview, which incorporates denoised, baseline-subtracted, and color-separated data from QUISCA. This alignment procedure consists of manual operations that include the definition of the range of interest, setting the parameters for peak detection, and selection of the reference peaks. The mobilities of sample peaks are then calibrated by the same algorithm as described for QUISCA.

\section{Peak Quantification}

Peaks selected by the user are quantified by their peak heights. The information on the heights and positions of selected peaks are exported from QUISCAview to an external file in spreadsheet format.

\section{User Interface}

Users can set several parameters for the operation of QUISCA as command line options. There is a standard (default) set of parameters that works well for most data presented to the program. These parameters can be further opti- mized to increase performance on data from any particular apparatus. QUISCAview is a graphical user interface of QUISCA that provides a global view of the processed data and allows users to align locally for allele calling. QUISCAview runs on computers equipped with Perl/Tk.

\section{RESULTS}

\section{Noise Filtering}

To apply the filter described above, the user can choose the parameter for frequency cut-offs so that useful signal information is not lost due to over-filtering. We tested the effect of the level of noise filtering by setting parameter $\mathrm{D}$ from $1 / \mathrm{m}$ to $20 / \mathrm{m}$, where $\mathrm{m}$ is a minimum integer power of two that is greater than the total time points of the run (9). A typical value for $m$ was 16384 using either instrument. In our environment, denoised data that is appropriate for the precise peak quantification without the incorrect splitting of the peaks or overestimation of baseline noise were empirically obtained to be at $\mathrm{D}=9 / \mathrm{m}$. Figure 2, panels $\mathrm{G}-\mathrm{I}$, shows that differences between the filtered signal and the original signal are not homogeneous at or near the peaks, especially in data from MegaBACE, which suggests that either the noise with heteroscedasticity (7) was removed or signal components were somewhat damaged. However, the differences associated with the peaks do not exceed a few percent of the peak heights and are unlikely to significantly disturb the quantification of the peaks.

\section{Baseline Subtraction}

After denoising with FFT, low-frequency variations of the baseline remain. In the current baseline correction method, the window size $\mathrm{N}$ is the only parameter amenable to optimization. $\mathrm{N}$ must be set larger than the width of any of the expected peaks to avoid undue reduction of peak heights but small enough to capture the characteristics of baseline drift. We found that the method worked well at $\mathrm{N}=500$, for a wide variety of data sets obtained from all tested instruments, as shown in Figure 2, panels J-L. 


\section{BioComputing/Biolnformatics > > > > >}

\section{Alignment (Calibration of Peak Po- sitions between Runs or Capillaries)}

Correct assignment of the peaks of the internal marker is vital for run-torun mobility calibration. To evaluate the peak assignment procedure, we created a peak set that contained 4783 peaks obtained from 168 runs of the GS500 TAMRA size standard (Applied Biosystems) on the ABI PRISM 3100 Genetic Analyzer. The choice of parameters for the assignment was made in a trial-and-error manner, and satisfactory results were achieved using $r=150$ and $\mathrm{R}=100$ (see Materials and Methods for these parameters). At these settings, we found that $98.1 \% \quad$ (4690 peaks) was correctly identified in the global alignment mode. We expect an improvement in the assignment accuracy by incorporating extra information available on each peak (e.g., peak shape and full width at half maximum, rather than peak heights and peak positions alone). Alternatively and perhaps more practically, one can use an internal marker that produces cleaner peaks in the SSCP conditions than the standard employed here. In the local alignment mode using QUISCAview, the reference peaks were manually assigned, and the alignments were always satisfactory (Figure 3).

\section{Quantitation}

Quantitative allele detection by PLACE-SSCP has been tested by mixing experiments (12). We compared the data processed by QUISCA and those processed by GeneScan ${ }^{\circledR}$ Analysis Software (Applied Biosystems), using the same data and method described previously by Sasaki et al. (12). Briefly,

(A)

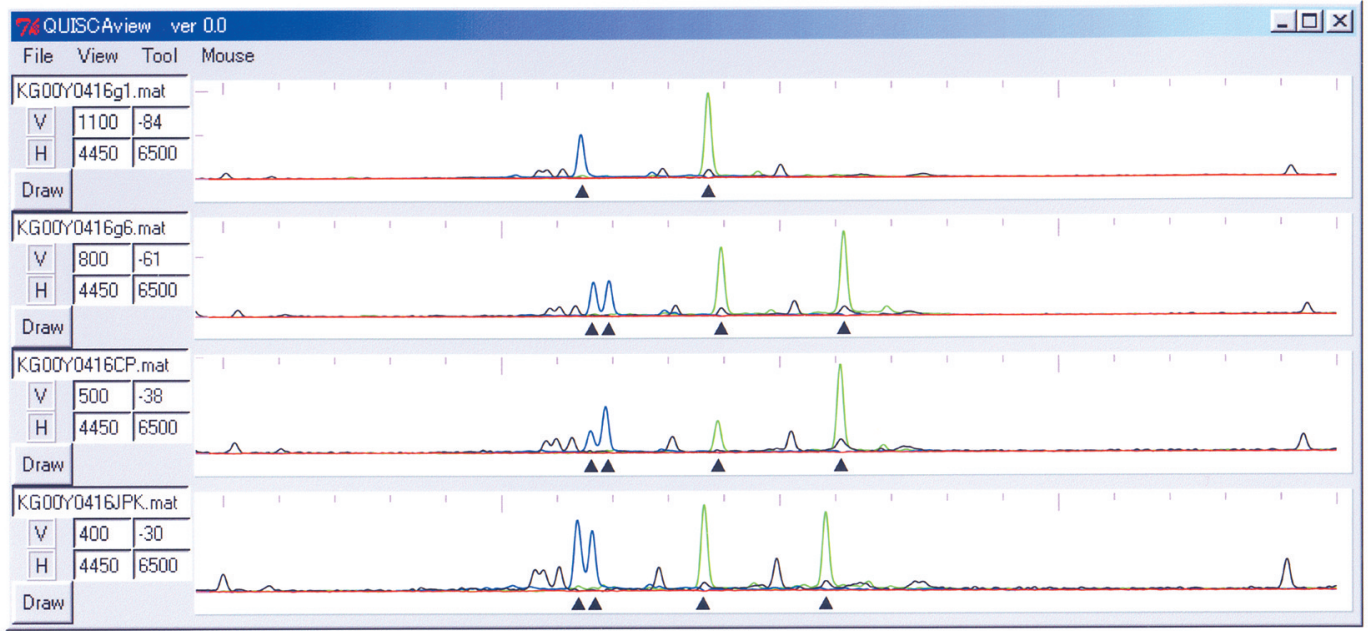

(B)

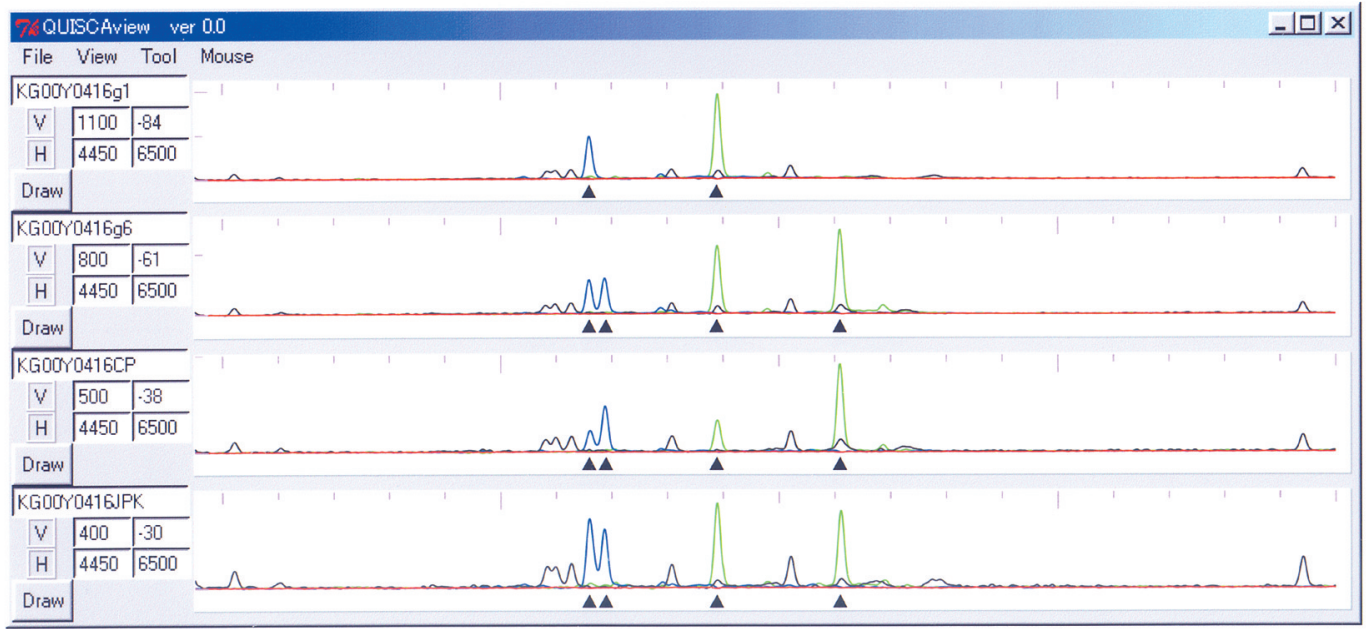

Figure 3. Chromatograms of PLACE-SSCP data before (A) and after (B) alignment. A sequence-tagged site containing an SNP (rs460752) was amplified from the DNA of two individuals (Japanese) and two pooled DNAs (Japanese pool and Centre d'Etude Polymorphisme Humaine pool) using forward (5'ATTCATTGCCCTATTTCAA-3') and reverse (5'-GTTAGCAAAGGATTGAATG-3') primers, followed by PLACE-SSCP analysis. Green and blue peaks were derived from the two complementary strands of the PCR product and differentially labeled with two fluorescent dyes (R110 and R6G, respectively). Reference peaks are in black. Arrowheads indicate the peak positions of the alleles. Capillary electrophoresis with an ABI PRISM 3100 Genetic Analyzer was performed in $12 \%$ linear polydimethylacrylamide buffered with $2 \times \mathrm{TME}(60 \mathrm{mM}$ Tris, $70 \mathrm{mM} 2$-morpholinoethanesulfonic acid, and $2 \mathrm{mM}$ EDTA, pH 6.8$)$ at $30^{\circ} \mathrm{C}$ as previously described (6) 
genomic DNAs of two individuals, who were homozygous for alternative alleles of a SNP, were combined at various ratios (from 0:10 to 10:0) and analyzed by PLACE-SSCP. Peak heights were quantified and normalized using the peak heights at the 1:1 ratio to correct PCR-bias. Table 1 shows that the calculated allele composition was in excellent agreement with the mixing ratios for the whole range. The coefficients of determinations $\left(\mathrm{r}^{2}\right)$ of mixing data processed by QUISCA and GeneScan were 0.999803 and 0.999729 , respectively. Thus, the current estimation (by QUISCA) was slightly better than the previous one, although the difference was marginal.

\section{DISCUSSION}

Fluorescence-based capillary gel electrophoresis has matured to become a technology capable of high-throughput DNA fragment analysis. With increasing availability of the instruments from various sources, a universal measure for reducing human intervention in data handling has become an issue. We have developed a program, QUISCA, with which multiple trace data of SSCP analysis obtained from various capillary array electrophoresis apparatus can be semi-automatically processed. QUISCA is also capable of handling the trace data of multi-dye, multi-injection experiments (data not shown).

The software supplied with some apparatus is inflexible and unsuitable for use in applications other than that specified, which does not include SSCP. In addition, we have sometimes experienced obvious over-filtering in the smoothing process with commercially available software that may be problematic for peak quantification. Therefore, we started to develop a program that is suitable for easy and flexible data collection and processing, without sacrificing accuracy in peak quantification. QUISCA allows one to perform rapid fragment analyses, not only genotyping and allele-frequency estimation of SNPs but also fragment sizing (data not shown).

QUISCA has been used extensively in a high-volume SNP detection and quantification project being carried out in our laboratory, which involves PLACE-SSCP analysis using ABI PRISM 3100 and 3700 Analyzers and MegaBACE. In this project, the software has been tested with the analysis of more than two thousand loci in approximately 10 individuals, which led to the identification of several hundred SNPs and estimations of their allele frequencies in populations using a pooled DNA strategy (Baba et al., manuscript in preparation). During these analyses, the software has evolved, and the parameters have been optimized. 
There are six parameters that users can use to modulate the performance of QUISCA. These parameters include: the determinant of high-frequency cutoff (D); window size in background estimation $(\mathrm{N})$; block margin and search range in block identification ( $\mathrm{r}$ and $\mathrm{R}$, respectively); and thresholds of height and width in peak detection ( $\mathrm{h}$ and $\mathrm{w}$, respectively). The default setting of these parameters are optimized for the multiple data obtained under a variety of conditions and with various apparatus (6), and we expect that the software will work sufficiently under other conditions of multi-capillary electrophoresis with no change in settings required.

Further improvements to this software are conceivable. Peaks may be more precisely quantified by improving the algorithms for noise removal and baseline subtraction. FFT is not efficient for the simultaneous correction of local and global changes in the signal because of the global character of the sine- and cosine-based function. Wavelet transform is one of the possible alternatives because of its functional property [i.e., that both time and frequency characteristics of the signal are captured $(8,16)]$. The second improvement should be to optimize the global alignment procedure. Currently, the assignment of peaks in a global alignment is not perfect and often requires local alignment, which involves human intervention. A potentially feasible strategy may be to integrate a multivariate analysis, in which relative peak heights, widths, positions, and shapes are considered statistically. Another possibility is the improvement of the assignment algorithm implemented in the current QUISCA (e.g., by taking dilation and other translation factors into consideration to find the most similar blocks between sample and template runs). QUISCA is currently being improved for further automation and precise peak identification and quantification.

Typically, in fluorescence-based gel electrophoresis, data undergo several processing steps before interpretation. There are some recent publications on software that can be used to perform automatic processing of the trace data for sequencing $(1,2)$. However, to our knowledge, software that flexibly processes data for fragment analysis such as peak quantification is scarce.
QUISCA can process SSCP data batch by batch, reference peaks are semi-automatically assigned at a high accuracy, and alleles can be precisely quantified. In the this version, the final decision of allele calling still requires human intervention. Additional enhancement of the software for automated allele calling and decision of presence/absence of SNPs is being developed. QUISCA is easily integrated into laboratory information management systems using its source code because the main body of QUISCA and its graphical user interface, QUISCAview, can be separated.

QUISCA is written in C and designed for cross-platform use, currently allowing the software to run in $\mathrm{Mi}$ crosoft ${ }^{\circledR}$ Windows ${ }^{\circledR}$ 95/98/2000, Linux, and UNIX. The QUISCAview software is also able to run under these operating systems provided that Perl (Perl5 or later) and the Perl/Tk module are installed. Supplementary information and updates can be found on our Web site at http://www.gen.kyushuu.ac.jp/ genome/quisca.html.

\section{ACKNOWLEDGMENTS}

This work was supported by a Grantin-Aid for Genome Science from the Ministry of Education, Culture, Sports, Science, and Technology, Japan. The authors thank other members of the Division of Genome Analysis, Research Center for Genetic Information, Medical Institute of Bioregulation, Kyushu University, for their constant cooperation and encouragement.

\section{REFERENCES}

1.Giddings, M.C., R.L. Brumley, Jr., M. Haker, and L.M. Smith. 1993. An adaptive, object oriented strategy for base calling in DNA sequence analysis. Nucleic Acids Res. 21:45304540 .

2.Giddings, M.C., J. Severin, M. Westphall, J. Wu, and L.M. Smith. 1998. A software system for data analysis in automated DNA sequencing. Genome Res. 8:644-665.

3.Inazuka, M., T. Tahira, and K. Hayashi. 1996. One-tube post-PCR fluorescent labeling of DNA fragments. Genome Res. 6:551-557.

4.Inazuka, M., H.M. Wenz, M. Sakabe, T. Tahira, and K. Hayashi. 1997. A streamlined mutation detection system: multicolor postPCR fluorescence labeling and single-strand conformational polymorphism analysis by capillary electrophoresis. Genome Res. 7:1094-1103.

5.Kruglyak, L. 1999. Prospects for wholegenome linkage disequilibrium mapping of common disease genes. Nat. Genet. 22:139144.

6.Kukita, Y., K. Higasa, S. Baba, M. Nakamura, S. Manago, A. Suzuki, T. Tahira, and K. Hayashi. 2002. A single-strand conformation polymorphism method for the large-scale analysis of mutations/polymorphisms using capillary-array electrophoresis. Electrophoresis 23:2259-2266.

7.Mittermayr, C.R., E. Rosenberg, and M. Grasserbauer. 1997. Detection and estimation of heteroscedastic noise by means of the wavelet transform. Anal. Commun. 34:73-75.

8.Perrin, C., B. Walczak, and D.L. Massart. 2001. The use of wavelets for signal denoising in capillary electrophoresis. Anal. Chem. 73:4903-4917.

9.Press, W.H., S.A. Teukolsky, W.T. Vetterling, and B.P. Flannery. 1988. Numerical Recipes in C. Cambridge University Press, Cambridge, UK.

10.Reich, D.E., M. Cargill, S. Bolk, J. Ireland, P.C. Sabeti, D.J. Richter, T. Lavery, R. Kouyoumjian, et al. 2001. Linkage disequilibrium in the human genome. Nature 411:199-204.

11.Sachidanandam, R., D. Weissman, S.C. Schmidt, J.M. Kakol, L.D. Stein, G. Marth, S. Sherry, J.C. Mullikin, et al. 2001. A map of human genome sequence variation containing 1.42 million single nucleotide polymorphisms. Nature 409:928-933.

12.Sasaki, T., T. Tahira, A. Suzuki, K. Higasa, Y. Kukita, S. Baba, and K. Hayashi. 2001. Precise estimation of allele frequencies of single-nucleotide polymorphisms by a quantitative SSCP analysis of pooled DNA. Am. J. Hum. Genet. 68:214-218.

13.Savitsky, A. and M.J.E. Golay. 1964. Smoothing and differentiation of data by simplified least squares. Anal. Chem. 36:16271639.

14.Southern, E.M. 1979. Measurement of DNA length by gel electrophoresis. Anal. Biochem. 100:319-323.

15.Venter, J.C., M.D. Adams, E.W. Myers, P.W. Li, R.J. Mural, G.G. Sutton, H.O. Smith, M. Yandell, et al. 2001. The sequence of the human genome. Science 291:1304-1351.

16.Workman, J., Jr., D.J. Veltkamp, S. Doherty, B.B. Andersen, M. Koch, J.F. Tatera, A.L. Robinson, L. Bond, et al. 1999. Process analytical chemistry. Anal. Chem. 71:121-180.

Received 31 May 2002; accepted 8 August 2002.

Address correspondence to:

Dr. Kenshi Hayashi

Division of Genome Analysis

Research Center for Genetic Information

Medical Institute of Bioregulation

Kyushu University

Maidashi 3-1-1, Higashi-ku

Fukuoka 812-8582, Japan

e-mail:khayashi@gen.kyushu-u.ac.jp 